Acta Cryst. (2002). A58 (Supplement), C175

CaO B1-B2 TRANSITION UNDER HIGH-PRESSURE AND TEMPERATURE

T. Yamanaka T. Nagai K. Kittaka

Graduate School of Science Osaka University Department of Earth and Space

Science 1-1 Machikaneyama TOYONAKA OSAKA 560-0043 JAPAN

X-ray powder diffraction measurements of $\mathrm{CaO}$ at high pressure and temperature have been performed with elevating temperature and pressure using lever-spring type diamond anvil cell with external ring heater. The diffraction patterns were collected by two-dimensional imaging plate (IP) detector (Rigaku R-AXIS IV++) having large dynamic range and high quantum efficiency. B1 structure transformed B2 at about $61.2-63.2 \mathrm{GPa}$ at room temperature in the process of increasing pressure and the back transformation from $\mathrm{B} 2$ to $\mathrm{B} 1$ phase at $59.8 \mathrm{GPa}$ in the course of depression. Aback transformation from B2 to B1 structure in the process of depression shows a large hysteresis.

External ring heater of $\mathrm{Pt}-\mathrm{Rh}(40)$ with $\mathrm{ZrO} 2-\mathrm{CaO}$ cement thermal-insulator was installed closely around DAC in the high-pressure generator. Temperature was regulated by PID controller and measured by thermocouple which contacts on diamond surface. Load pressure was cooled by the newly devised remote system with oil pressure control. Water circulation in the high-pressure system was attached. B1-B2 transition pressure becomes slightly lower from 58.8GPa at $300 \mathrm{~K}$ to $53.1(\mathrm{GPa})$ at $685 \mathrm{~K}$ indicating $\mathrm{dP} / \mathrm{dT}<0$. But $\mathrm{B} 1-\mathrm{B} 2$ transition is highly dependent on pressure instead of temperature. From the viewpoint of relative volume between $\mathrm{B} 1$ and $\mathrm{B} 2, \mathrm{CaSiO}_{3}$ perovskite is possibly decomposed to $\mathrm{SiO}_{2}\left(\mathrm{CaCl}_{2}\right.$ type $)$ and $\mathrm{CaO}-\mathrm{B} 2$ structure in the pressure and temperature range of lower mantle.

Keywords: CaO B1-B2 TRANSITION HIGH PRESSURE

Acta Cryst. (2002). A58 (Supplement), C175

\section{SEARCH FOR INTERSTITIAL ELECTRONS IN THE STRUCTURE} OF Cs-IV

C. Hejny M.I. McMahon R.J. Nelmes

The University of Edinburgh Department of Physics and Astronomy King's Buildings Mayfield Road EDINBURGH EH9 3JZ EH9 3JZ UK

The high-pressure behavior of $\mathrm{Cs}$ is believed to result from pressure-driven sto-d electron transfer, which results in a number of structural phase transitions and unusual optical and melting behavior. In addition, the s-to-d transition changes the bonding character of $\mathrm{Cs}$ from the non-directional bonding of a nearly-free electron metal at ambient pressure to more complex directional bonding at high pressures. As a result, Cs transforms from simple structures to more complex open-packed structures on pressure increase.

Calculations of the electron density in one of these complex phases, Cs-IV [1, 2], stable between $4.5 \mathrm{GPa}$ and $10 \mathrm{GPa}$, and in the predicted high-pressure complex phases of $\mathrm{Li}$ [3] and $\mathrm{Na}$ [4], suggest that in each structure there should be interstitial electron density between the atomic sites. Indeed, it may be possible to regard Cs-IV as 'Cesium electride' $\mathrm{Cs}^{+} \mathrm{e}$ '. To investigate this, we have made detailed $\mathrm{x}$-ray diffraction studies of Cs-IV using high-quality single crystals and synchrotron radiation.

References:

[1] Takemura, K., Minomura, S., Shimomura, O. (1982) Phys. Rev. Lett. 49, $1772-1775$

[2] von Schnering, G., Nesper, R. (1987) Angew. Chem. Int. Edn. Engl. 26, $1059-1200$

[3] Neaton, J.B., Ashcroft, N.W. (1999) Nature 400, 141 - 144

[4] Neaton, J.B., Ashcroft, N.W. (2001) Phys. Rev. Lett. 86, 2830 - 2833

Keywords: HIGH PRESSURE, CRYSTAL STRUCTURE, ELECTRON DENSITY
Acta Cryst. (2002). A58 (Supplement), C175

ISOTOPE EFFECTS ON COMPRESSIBILITIES AND PRESSURE

DEPENDENCY OF CAGE FILLING IN METHANE CLATHRATE HYDRATES

E. Goreshnik A. Klapproth H. Klein W.F. Kuhs

GZG Crystallography, University Goettingen Goldschmidtstr. 1 GOETTINGEN 37077 GERMANY

Methane hydrates are found in ocean sea floor sediments and in permafrost regions attracting considerable interest during last years as giant source of natural gas and as a potential geo hazard. Here we report on the first in situ structural work on hydrogenated and deuterated methane hydrates. The samples were investigated at $\mathrm{T}=271 \mathrm{~K}$ in the pressure range of $50-1000 \mathrm{bar}$ at neutron and synchrotron sources (D2B at ILL, ID15A at ESRF and BW5 at HASYLAB. All data were analyzed using a Rietveld-refinement procedure implemented in the program GSAS. Final R-factors 2-3\% were achieved. The pressure dependent lattice constants were obtained with a least-squaresestimated precision of $0.0025-0.0075 \%$. The isotope effect is larger in the hydrates $(0.15 \%)$ than in hydrogenated and deuterated ice Ih $(0.08 \%)$. Bulk moduli for hydrogenous samples $(9.11 \mathrm{GPa})$, established on ESRF and HASYLAB data sets, were found essentially the same, and rather differ from deuterated samples bulk modulus value $8.21 \mathrm{GPa}$, based on neutron and X-ray synchrotron data sets. The values of cage filling were established on the ESRF data set. The large cages are almost fully occupied at all pressures, while the filling of small cages increases with pressure but less then expected from a solid solution behavior. In contrast to all predictions the small cage data cannot be fitted by a Langmuir isotherm.

Keywords: GAS HYDRATES COMPRESSIBILITY ISOTOPE EFFECT

\section{Acta Cryst. (2002). A58 (Supplement), C175}

\section{HIGH PRESSURE STRUCTURAL STUDIES ON GALLIUM}

\section{O. Degtyareva M.I. McMahon R.J. Nelmes}

University of Edinburgh Department of Physics and Astronomy James Clerk Maxwell Building, The King's Buildings, Mayfield Road EDINBURGH EH9 $3 \mathrm{JZ} \mathrm{UK}$

Gallium, a group III element, exhibits a complex P-T phase diagram with a number of metastable phases. On pressure increase at room temperature Ga melts and around $2 \mathrm{GPa}$ crystallizes in the metastable Ga-III phase which has an In-type structure (body-centered tetragonal with 2 atoms) [1]. This phase has to be supercooled down to $-63^{\circ}$ to $-88^{\circ} \mathrm{C}$ at around $3 \mathrm{GPa}$ before it transforms to the stable Ga-II phase. The structure of Ga-II has been reported to be body-centered cubic with 12 atoms in the unit cell (cI12) [1]. However, diffraction studies on $\mathrm{Ga}$ [2] between 3 and $15 \mathrm{GPa}$ showed complex patterns, that could not be indexed with the cI12 structure. Above $15 \mathrm{GPa}$ at room temperature, Ga-II is reported to transform to Ga-III, which is the stable phase at this pressure [2], and then to a fcc phase, Ga-IV, at $120 \mathrm{GPa}$ [3]. To help identify the outstanding uncertainties with the gallium P-T phase diagram, we have undertaken detailed structural studies of $\mathrm{Ga}$ up to $20 \mathrm{GPa}$ at room temperature and at temperatures down to $-80^{\circ} \mathrm{C}$ at 3-5 $\mathrm{GPa}$. These combined powder and single-crystal $\mathrm{x}$-ray diffraction studies show new features in the P$\mathrm{T}$ phase diagram, including two phases with complex crystal structures that have not been reported previously.

References

[1] L. Bosio (1978). J. Chem. Phys. 68(3), 1221-1223; and references therein.

[2] O. Schulte and W.B. Holzapfel (1997). Phys. Rev. B 55, 8122-8128.

[3] K.Takemura, K.Kobayashi, and Arai Masao (1998). Phys. Rev. B 58, $2482-$ 2486.

Keywords: HIGH PRESSURE, CRYSTAL STRUCTURES, PHASE TRANSITIONS 